\title{
Global DNA methylation and transcriptional analyses of human ESC-derived cardiomyocytes
}

\author{
Ying Gu${ }^{1}$, Guang-Hui Liu ${ }^{2,3 \bowtie}$, Nongluk Plongthongkum ${ }^{4}$, Christopher Benner ${ }^{1}$, Fei $\mathrm{Yi}^{1}$, Jing $\mathbf{Q u}^{2}$, \\ Keiichiro Suzuki ${ }^{1}$, Jiping Yang ${ }^{2}$, Weiqi Zhang ${ }^{2}, \mathrm{Mo} \mathrm{Li}^{1}$, Nuria Montserrat ${ }^{5,6}$, Isaac Crespo ${ }^{7}$, Antonio del Sol ${ }^{7}$, \\ Concepcion Rodriguez Esteban ${ }^{1}$, Kun Zhang ${ }^{4 凶}$, Juan Carlos Izpisua Belmonte ${ }^{1,5 \bowtie}$ \\ ${ }^{1}$ Gene Expression Laboratory, Salk Institute for Biological Studies, 10010 North Torrey Pines Road, La Jolla, CA 92037, USA \\ ${ }^{2}$ National Laboratory of Biomacromolecules, Institute of Biophysics, Chinese Academy of Sciences, Beijing 100101, China \\ ${ }^{3}$ State key Laboratory of Drug Research, Shanghai Institute of Materia Medica, Chinese Academy of Sciences, \\ Shanghai 201203, China \\ ${ }^{4}$ Department of Bioengineering, University of California at San Diego, La Jolla, CA 92093, USA \\ ${ }^{5}$ Center of Regenerative Medicine in Barcelona, Dr. Aiguader 88, 08003 Barcelona, Spain \\ ${ }^{6}$ Biomedical Research Networking Center in Bioengineering, Biomaterials and Nanomedicine (CIBER-BBN), Poeta Mariano \\ Esquillor s/n, 50018 Sargossa, Spain \\ 7 Luxembourg Centre for Systems Biomedicine (LCSB), University of Luxembourg, Walferdange, Luxembourg \\ $\square$ Correspondence: ghliu@ibp.ac.cn (G.-H. Liu), kzhang@bioeng.ucsd.edu (K. Zhang), belmonte@salk.edu, \\ izpisua@cmrb.eu (J. C. Izpisua Belmonte)
}

Received July 1, 2013 Accepted July 30, 2013

\begin{abstract}
With defined culture protocol, human embryonic stem cells (hESCs) are able to generate cardiomyocytes in vitro, therefore providing a great model for human heart development, and holding great potential for cardiac disease therapies. In this study, we successfully generated a highly pure population of human cardiomyocytes (hCMs) (>95\% c $\mathrm{TnT}^{+}$) from hESC line, which enabled us to identify and characterize an hCM-specific signature, at both the gene expression and DNA methylation levels. Gene functional association network and gene-disease network analyses of these hCM-enriched genes provide new insights into the mechanisms of hCM transcriptional regulation, and stand as an informative and rich resource for investigating cardiac gene functions and disease mechanisms. Moreover, we show that cardiac-structural genes and cardiac-transcription factors have distinct epigenetic mechanisms to regulate their gene expression, providing a better understanding
\end{abstract}

Electronic supplementary material The online version of this article (doi:10.1007/s13238-013-0016-x) contains supplementary material, which is available to authorized users. of how the epigenetic machinery coordinates to regulate gene expression in different cell types.

KEYWORDS human cardiomyocyte, DNA methylation, microarray, heart development

\section{INTRODUCTION}

Heart failure, caused by massive loss or dysfunction of hCMs, is the main cause of death and morbidity in the developed world. Treatments for this devastating disorder are inefficient and usually focused around symptomatic alleviation, while the main cause of the disease, that is, loss of hCMs and associated contractile function, remains unchallenged. Thus, identification of factors reducing fibrotic scarring or promoting $\mathrm{hCM}$ proliferation is of the utmost importance for public health. Achieving this goal is complicated by the difficulty of obtaining large numbers of pure, fully differentiated hCMs and their intermediate stages.

hESCs have the potential to differentiate into cells of all lineages, therefore providing an ideal in vitro model to study organ development and disease mechanisms. Recent efforts have successfully developed several protocols, by which hESCs can be differentiated into hCMs (Yang et al., 2008; Kattman et al., 2011; Lian et al., 2012; Willems et al., 
A
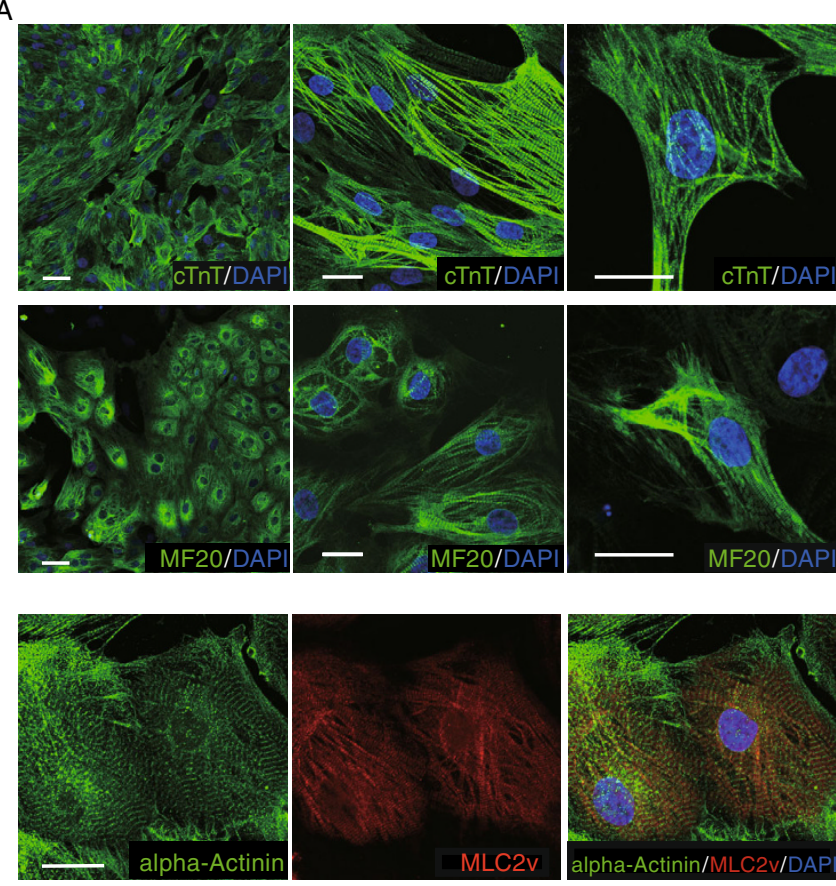

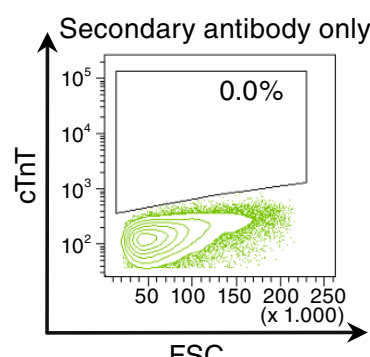

FSC
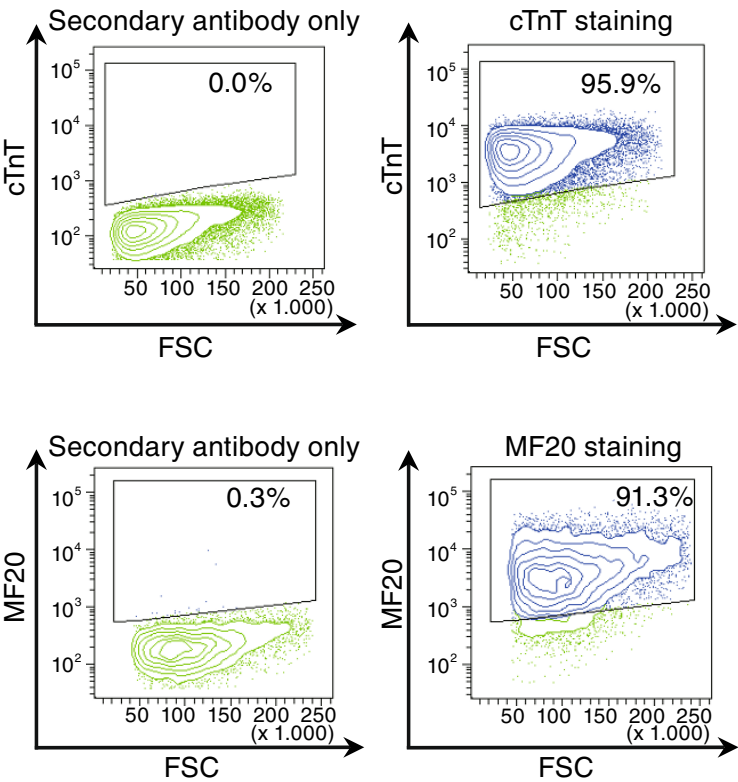

Figure 1. Characterization of hESC-derived hCMs. (A) Immunofluorescence analyses showing the expression of key cardiac markers in d25 hCMs derived from H9 hESCs. Top panel: cTnT (green). Middle panel: MF20 (green). Bottom Panel: alpha-Actinin (green) and MLC-2v (red). Scale bar: $20 \mu \mathrm{m}$. (B) Flow cytometry analysis of cells expressing cTnT (top panel) or MF20 (bottom panel). Cells were collected on day 25 of hCM differentiation.

2012; Zhang et al., 2012). Furthermore, hESC-derived hCMs have been analyzed by microarray and a group of cardiospecific genes were revealed (Beqqali et al., 2006; Cao et al., 2008; Synnergren et al., 2008). However, one inevitable concern is that by these published differentiation protocols, it is difficult to obtain pure hCMs. Hence, any following study using the mixed cell population may potentially give some misleading results. To overcome this, an improved protocol is needed in order to achieve a satisfactory purity of hESC-derived hCMs. Moreover, while the gene expression profile for hCMs has been provided, a more comprehensive investigation on gene expression together with epigenetic regulation, such as DNA methylation, during the $\mathrm{hCM}$ differentiation is still lacking.

In an effort to overcome these limitations, we have developed a new cardiac differentiation protocol starting from $\mathrm{hESCs}$ that yields a highly pure population of hCMs $(>95 \%)$ suitable for genomic studies. As proof-of-concept, and in an effort to uncover new cardiac-specific targets relevant for therapeutic applications, we performed global epigenetic and transcriptional analyses during cardiac specification using this protocol. We performed transcriptional profiling and genome-wide DNA methylation analyses of hCMs and compared them to undifferentiated hCMs and hESC-derived neural stem cells (hNSCs). Our results provide a step forward towards the characterization of hCMs at both the transcriptional and epigenetic levels, and offer a powerful tool towards better understanding heart physiology and disease.

\section{RESULTS}

\section{Derivation of highly enriched cardiomyocytes from hESCs}

Following Palecek's previous protocol (Lian et al., 2012), hESCs were seeded as single cells on Matrigel and maintained in mTeSR. The GSK3 specific inhibitor CHIR99021 was added on the first day of differentiation, followed by the Wnt inhibitor IWP4 on day 3 . After 15 days, a relatively pure and contracting cardiomyocyte population was obtained (Movie S1). We enriched this fraction by collecting and washing the contracting hCM sheets and re-plating them on fresh Matrigel plates (Movies S2 and S3). These subcultured hCMs expressed the CM-specific markers cardiac troponin T (cTnT) and sarcomeric myosin (MF20), and exhibited normal cardiac sarcomere organization, as indicated by alpha-Actinin and MLC2v co-staining (Fig. 1A). Flow cytometry analysis indicated a majority of definitive cardiac cells were present at day $25\left(96 \% \mathrm{cTnT}^{+}\right.$cells and $91 \% \mathrm{MF}^{+} 0^{+}$, Fig. 1B).

\section{Global gene expression profiling in hCMs}

We obtained RNA from undifferentiated hESCs and hCMs and used it for microarray analysis. hESC-derived hNSCs 
A

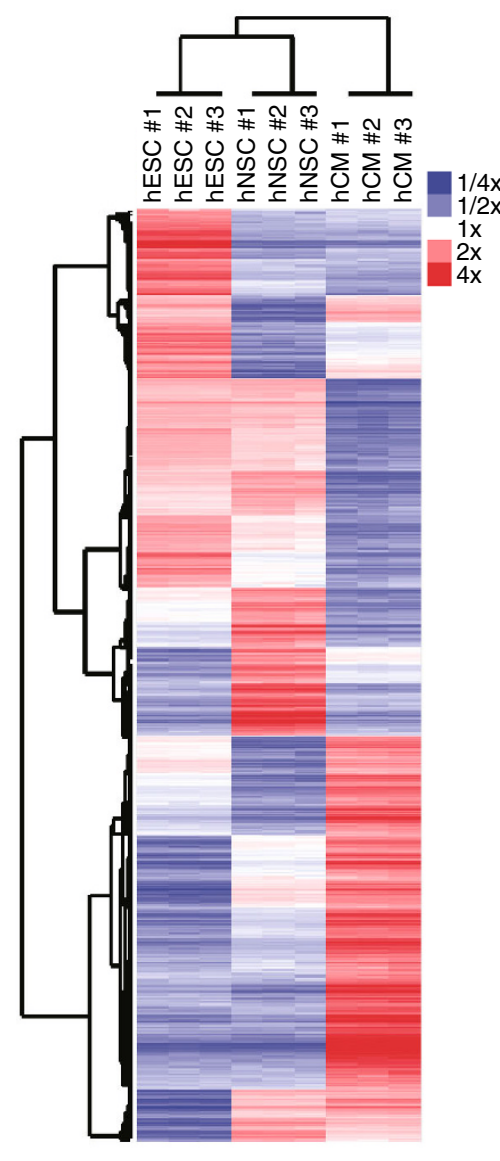

B

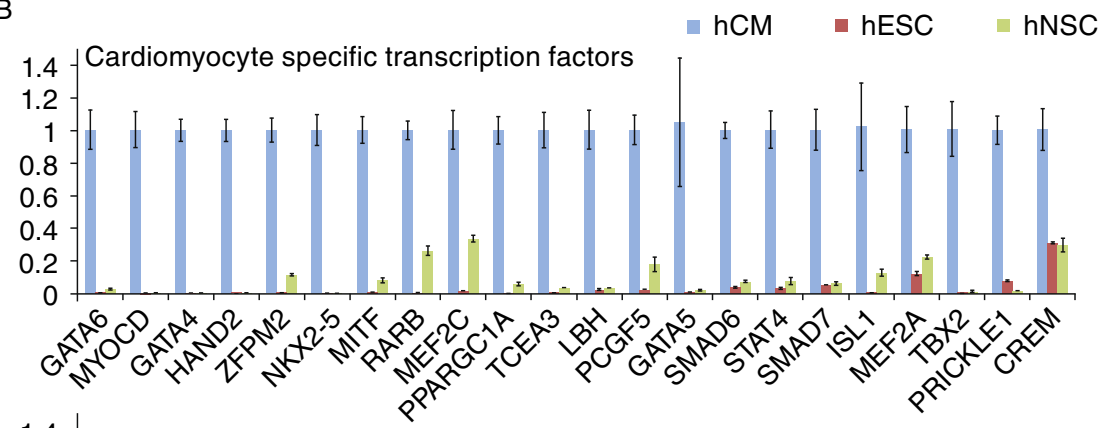

1.4 Cardiac disease related genes
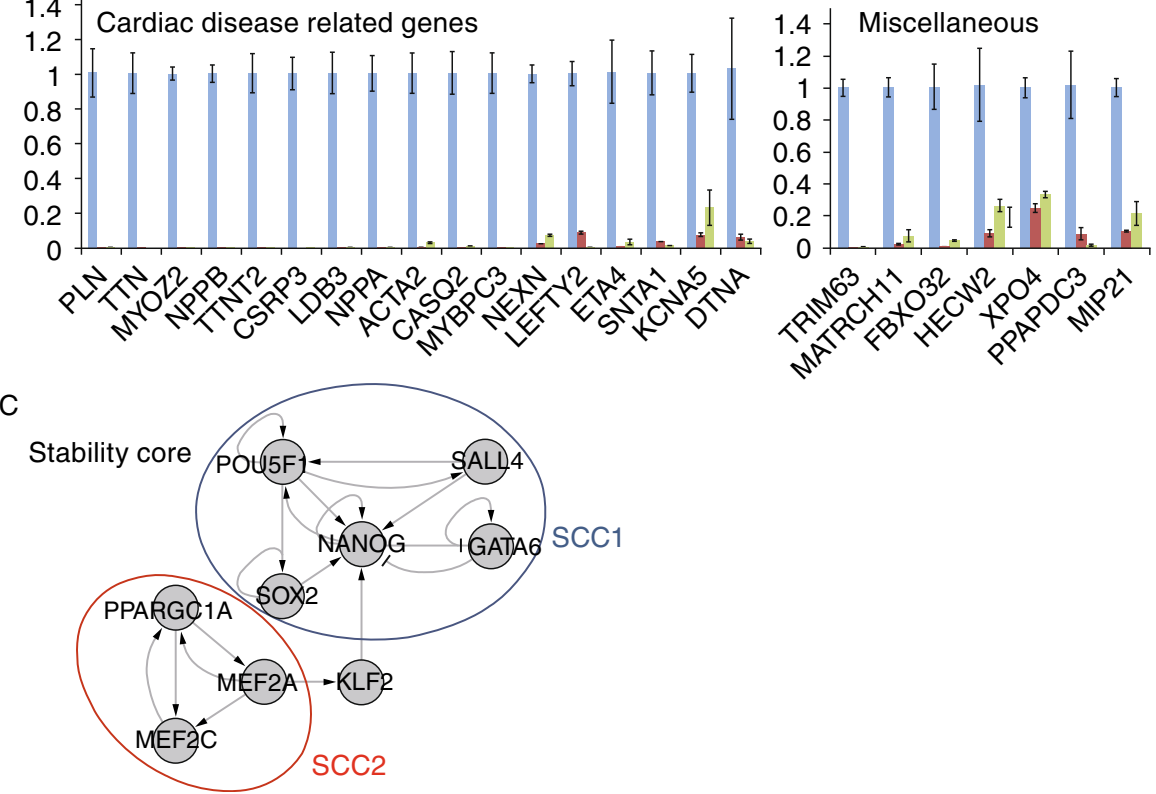

Figure 2. Global gene expression profiling of hCMs. (A) Heatmap and hierarchical clustering analysis of gene expression profiles of hESC, hNSCs, and hCMs performed in triplicate. Color represents the expression level relative to mean. (B) RT-qPCR analysis of transcript expression in hESCs, hCMs, and hNSCs. The expression levels of genes in hCMs were set to one. Data are shown as mean \pm s.d., $n=3$. (C) The gene regulation network that includes a stability core of 9 genes distributed in two strongly connected components (SCCs). These two SCCs or clusters of circuits, named as SCC1 and SCC2 could be broadly linked to pluripotency cellular features and the hCM-specific properties, respectively. Nodes represent genes and edges regulatory interactions positive (“ $\rightarrow$ ") and negative (“-|").

(Liu et al., 2012) were used as a control population. All the cells shared the same genetic background (H9), allowing for an unbiased side-by-side comparison of their gene expression profile. Three biological replicates from each cell type were measured with PrimeView Human Gene Expression Arrays, covering more than 36,000 transcripts and variants. All of the replicates were highly reproducible, supporting the purity and reliability of the method. Clustering data indicated that hESCs and hNSCs were closer to each other in terms of expression, while hCMs showed a more distinct expression pattern (Fig. 2A). Among represented transcripts, we identified 695 genes that showed at least a two-fold up-regulation and 401 genes that showed at least a two-fold down-regulation in hCMs compared to both hESCs and hNSCs (Tables S1 and S2). A group of the cardiac-enriched genes were validated by qRT-PCR (Fig. 2B).
To gain further insight into the functions of these hCM differentially expressed genes, we performed Gene Ontology (GO) analyses using the BiNGO (Maere et al., 2005) Cytoscape (Shannon et al., 2003) plugin. Interestingly, hCM upregulated genes were significantly over-represented in cardiac function-related GO terms (complete lists of GO terms are shown in Tables S3-5), including muscle contraction, heart development, and sarcomeric structures. In contrast, hCM differentially down-regulated genes were significantly clustered into $\mathrm{GO}$ terms such as $\mathrm{M}$ phase, nuclear division, and mitosis (complete lists of GO terms are shown in Tables S6-8), suggesting that mitosis in hCMs is strongly repressed, as has been consistently observed in hCMs during maturation (Zhang et al., 2012).

Next, we analyzed differentially regulated targets in the context of gene regulatory networks. We could identify the 
minimal combinations of reprogramming determinants responsible for the transition of hESCs towards hCMs. Specifically, our computational model defined a gene regulatory network stability core with two major components associated with both pluripotency and hCMs. Perturbation of these genes (up- or down-regulation, depending on the original state) triggered a regulatory chain reaction resulting on the transition of hESCs to hCMs (Fig. 2C).

Interaction network and gene-disease network analyses of hCM-enriched genes

To better understand the functional interaction between the identified hCM-specific genes, all of the $695 \mathrm{hCM}$-enriched genes were screened into GeneMANIA (Montojo et al., 2010) Cytoscape plugin to produce a functional association network based on their relationships, such as co-expression, co-localization, genetic interaction, and physical interaction (a complete information of the interactions is shown in Table S9). Subnetworks of functional associations between genes involved in muscle contraction, heart development and cardiac transcriptional regulation were also generated (Fig. 3, complete information of interactions are shown in Tables S10-12). These networks may help in describing new relationships and provide a systematic resource for cardiac gene function prediction.

Phenotype-genotype relationship studies facilitate the understanding of the normal and diseased physiology. We used the DisGeNET (Bauer-Mehren et al., 2010) plugin of Cytoscape to generate a gene-disease network of highly expressed hCM-specific genes. Of the top 50 up-regulated hCM-specific genes we analyzed, 27 of them (54\%) showed associations with at least one known cardiovascular diseases and 5 of them (NPPB, TNNT2, NPPA, RYR2, and PLN) were linked to more than 10 different types of cardiovascular diseases (Fig. 4A, Table S13). In addition, a total number of 142 disease-associated mutations were found in those top hCM-enriched genes (mutations listed in Fig. 4B, references listed in Table S14), suggesting a preferred enrichment of disease causing mutations in hCM-specific genes responsible for critical human heart functions.

\section{Genome-wide DNA methylation analysis of hCMs}

Up to date, relatively few studies have analyzed global DNA methylation status in human cardiac lineages. hESCs, hCMs, and hNSCs were collected for genome-wide DNA methylation profiling using methylation sequencing with bisulfite padlock probes as previously described (Diep et al., 2012). hCMs displayed the highest global DNA methylation level (Fig. 5A) and 985 genes showed an increase in their promoter $\mathrm{mCpG}$ levels (5\% or more), whereas 195 genes exhibited a $5 \%$ or more decrease of promoter DNA methylation compared to hESCs and hNSCs (Tables S15 and S16). Interestingly, these hCM-specific demethylated genes were significantly over-represented in our cardiac-related GO terms search, suggesting that promoter DNA demethylation may contribute to the regulation of cardiac functions (Tables S17-19). Since DNA methylation represents an essential epigenetic mechanism for gene repression, we speculated whether decreased DNA methylation correlated with the increased expression in cardiac-related genes. We combined DNA methylation and microarray data together and identified a group of 29 genes that exhibited hCM-specific promoter demethylation, as well as at least a two-fold upregulation in gene expression (Table S20). These genes were highly enriched in cardiac-structural related GO terms such as muscle contraction, structural muscle constituents, and sarcomere-related transcripts (Tables S21-23). Again, this data supported the notion that genes encoding cardiacstructural proteins are largely regulated by DNA methylation in hCMs. However, and since the majority of demethylated genes did not show significant changes in gene expression, other layers of regulation, such as histone modifications, might also play a significant role (Xie et al., 2013).

Among those cardiac-structural genes that were specifically demethylated in hCMs, a majority of them exhibited hypermethylated $\mathrm{mCpG}(>70 \%)$ at the promoter regions in hESC and hNSCs (Fig. 5B), indicating that DNA methylation in the promoter region of these genes may function as a major repressive signal in non-cardiac lineages. In contrast, cardiac-specific transcription factors (TFs), such as NKX2.5, GATA6, GATA4, MYOCD, HAND2, TBX5, and TBX18, were more expressed in hCMs than in hESCs or hNSCs, while displaying a similar pattern of low promoter $\mathrm{mCpG}$ levels $(<10 \%)$ in all three lineages, suggesting that the regulation of the expression of cardiac-specific TFs is largely independent of DNA methylation (Fig. 5B).

\section{DISCUSSION}

In this study, we report on an improved protocol for the generation of hCMs from hESCs, which enabled us to identify and characterize a hCM-specific signature, at both the gene expression and DNA methylation levels. The interaction networks we present in this study describe the precise mutual relationships between every hCM-enriched genes, as well as the critical genes involved in the fundamental heart functions, therefore providing an informative and systematic resource for gene function prediction in cardiac research field. In regard to understanding disease mechanisms, we identified a tight correlation between various heart diseases and hCM highly up-regulated genes. These findings not only highlight the importance of these cardiac-specific genes in maintaining normal heart development and functions, but may also provide novel gene targets for uncovering heart disease mechanisms.

By combining the promoter DNA methylation and gene expression profile, we got an overall glance at the gene regulation by DNA methylation in hCMs. Interestingly, we found that a group of cardiac-structural genes exhibited 


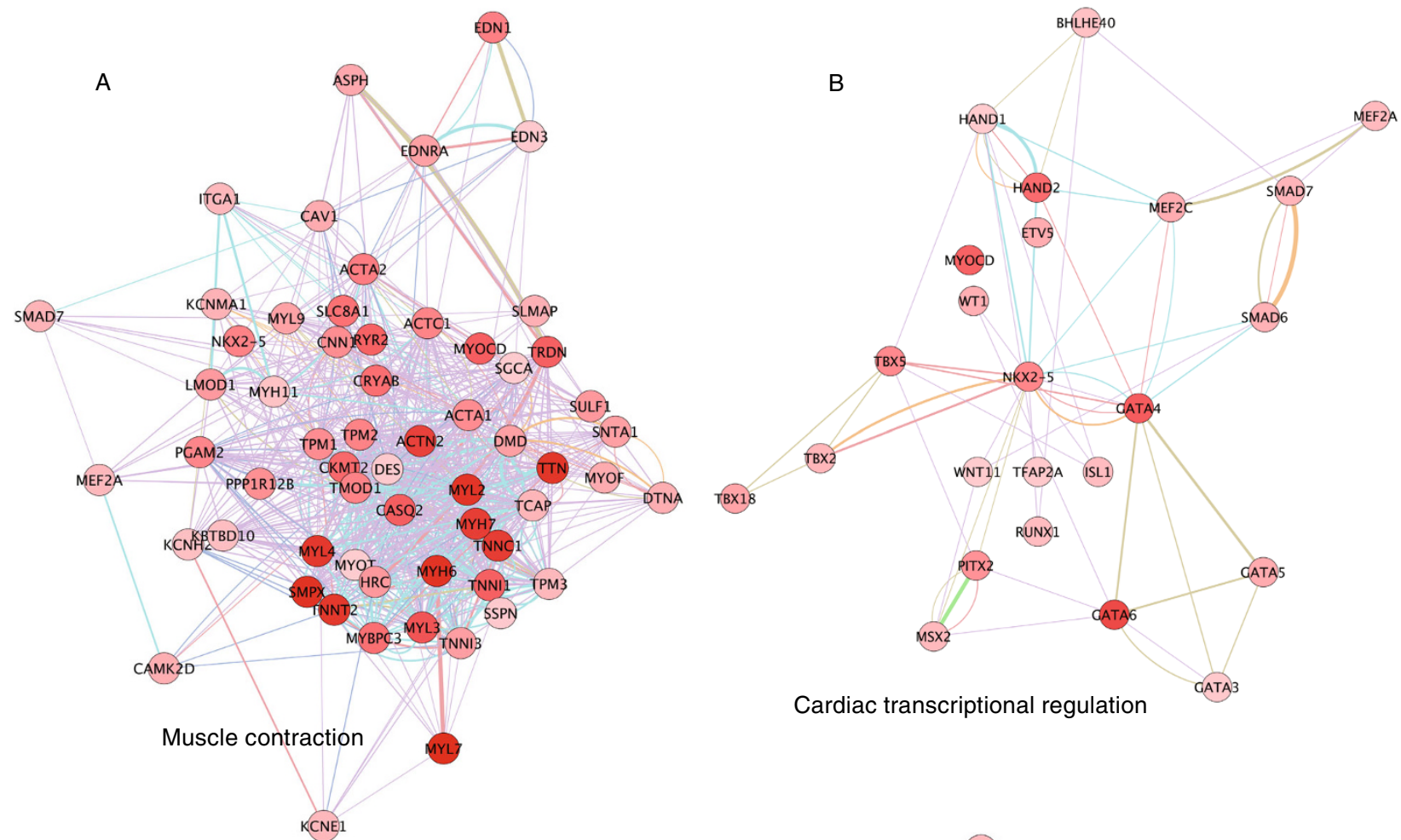

C

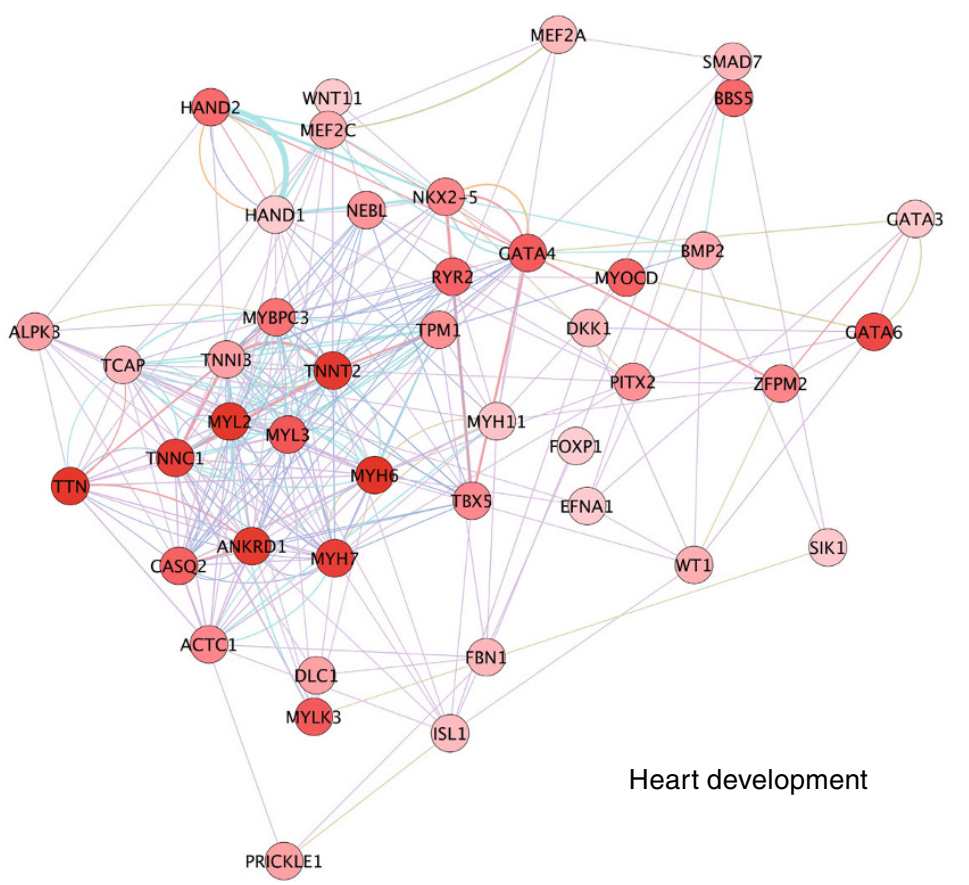

Figure 3. Association networks of hCM-enriched genes involved in function groups of muscle contraction, heart development, and cardiac transcriptional regulation. Networks were generated by GeneMANIA Cytoscape plugin. Nodes represent genes involved in the selected function group, and edges represent the relationships between linked genes. Node colors represent the average expression fold changes in hCMs compared to both hESCs and hNSCs.

hypermethylated $\mathrm{mCpG}$ at the promoter regions in hESCs and hNSCs, but were demethylated in hCMs, suggesting that promoter DNA methylation may be employed as a major transcription repression mechanism in these genes.
Supporting this notion, cardiac-structural genes were minimally expressed and often lacked another essential repressive mark, H3K27me3, in human ESCs and early stage cells of multiple non-cardiac lineages (Paige et al., 2012; Xie 
A

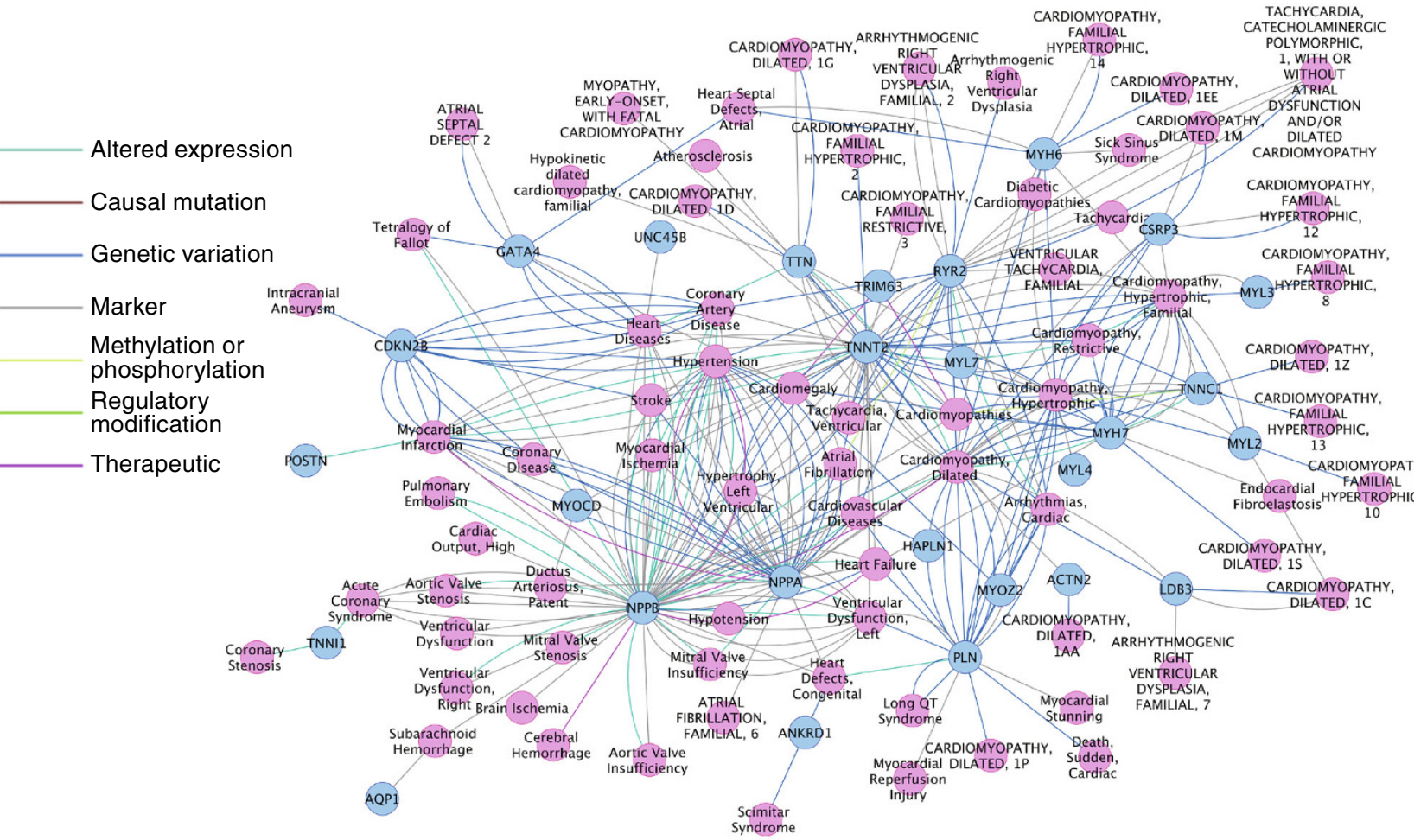

B

\begin{tabular}{|c|c|c|}
\hline Gene & Diseases & Mutations in DNA or protein sequence \\
\hline PL & $\begin{array}{l}\mathrm{HCM} \\
\mathrm{DCM}\end{array}$ & $\begin{array}{c}-42 \mathrm{C}>\mathrm{G},-77 \mathrm{~A}>\mathrm{G} \\
\mathrm{R} 9 \mathrm{C}, \mathrm{R} 14 \mathrm{del}\end{array}$ \\
\hline \multirow{2}{*}{ MYH6 } & \multirow{3}{*}{$\begin{array}{l}\text { HCM } \\
\text { or DCM } \\
\text { ASD } \\
\text { HCM }\end{array}$} & R795Q, P830L, A1004S, Q1065H, E1457K \\
\hline & & I820N \\
\hline MYOZ2 & & S48P, I246M \\
\hline MYL2 & $\mathrm{HCM}$ & $\begin{array}{l}\text { A13T, F18L, E22K, N47K, R58Q, K103E, } \\
\text { IVS6-1G>C }\end{array}$ \\
\hline CSRP3 & $\begin{array}{l}\text { HCM } \\
\text { DCM }\end{array}$ & $\begin{array}{c}\text { L44P, S54R/E55G, C58G, 172T>G } \\
\text { W4R }\end{array}$ \\
\hline \multirow{2}{*}{ TTN } & HCM & \multirow{2}{*}{$\begin{array}{c}\text { R740L, S3799Y } \\
\text { V54M, A743V, W930R, R25618Q, Q4053ter, } \\
\text { S4465D, 2-bp insertion in exon } 326\end{array}$} \\
\hline & DCM & \\
\hline LDB3 & $\begin{array}{l}\text { DCM } \\
\text { LVNC }\end{array}$ & $\begin{array}{c}\text { D117N, K136M, S196L, T213I, I352M, D626N } \\
\text { D117N, K136M, S196L, }\end{array}$ \\
\hline \multirow{3}{*}{ MYH7 } & DCM & $\begin{array}{l}\text { P211L, L390V, R403W, R403Q, Y501C, } \\
\text { H576R, R663C, Q734P, I736T, G741W, A797T, } \\
\text { R870H, A901G, D928N, E930K, R1053Q, } \\
\text { E1356K, A1379T, A1454T, R1500W, S1776G }\end{array}$ \\
\hline & DCM & $\begin{array}{l}\text { I201T, T412N, A550V, T1019N, R1193S, } \\
\text { E1426K, R1634S }\end{array}$ \\
\hline & LVNC & M531R \\
\hline NKTD & TAPVR & $10 ; 21$ translocation \\
\hline
\end{tabular}

\begin{tabular}{ccc} 
Gene & Diseases & Mutations in DNA or protein sequence \\
\hline \multirow{2}{*}{ NPPB } & HCM & D175N \\
& EH & Variable number of tandem repeat of \\
& DCM & Iflanking region \\
& ICM & 1741W, K210del
\end{tabular}

179N, 190M, R92C, R92W, F110I, K124N, R130C, R278C, G287A, sexon 14, a five-base
(CTTCT) insertion/deletion in intron 3

\begin{tabular}{|c|c|c|}
\hline & $\begin{array}{l}\text { Isehemic } \\
\text { Stroke }\end{array}$ & G66A \\
\hline NPPA & $\begin{array}{l}\text { AF } \\
\text { EH } \\
\text { LVH }\end{array}$ & $\begin{array}{c}\text { C. } 456-457 \text { delAA } \\
\text { V7M, }-664 G \\
-2843 A>G\end{array}$ \\
\hline TNNC1 & $\begin{array}{l}\mathrm{HCM} \\
\mathrm{DCM}\end{array}$ & $\begin{array}{c}\text { A8V, L29Q, A31S, C84Y, E134D, D145E } \\
\text { Y5H, M103l, D145E, I148V }\end{array}$ \\
\hline ACTN2 & DCM & Q9R \\
\hline MYL3 & $\mathrm{HCM}$ & D778E \\
\hline GATA4 & $\mathrm{CHD}$ & $\begin{array}{c}\text { A6V, S52F, P163S, E216D, E359K, S377G, } \\
\text { P407Q, S429T, A442V, IVS4 + 55C >A } \\
\text { c.1074delC }\end{array}$ \\
\hline RYR2 & CPVT & $\begin{array}{c}\text { R414C, E1724K, A2254V, R2267H, A2394G, } \\
\text { V2475F, F4020L, E4076K, N4104I, N4104K, } \\
\text { H4108N, H4108Q, R4497C, S4565R, G4662S, } \\
\text { H4762P, V4771l, P4902S, genomic deletion } \\
\text { involved RYR2 exon-3 }\end{array}$ \\
\hline MYOCD & $\mathrm{CHD}$ & K259R \\
\hline TRIM63 & HCM & A48V, I130M, Q247del \\
\hline
\end{tabular}

\begin{tabular}{ll}
\hline DCM & Dilated Cardiomyopathy \\
HCM & Hypertrophic Cardiomyopathy \\
RCM & Restrictive Cardiomyopathy \\
ASD & Atrial Septal Defect \\
LVNC & Left Ventricular Non-Compaction \\
TAPVR & Total Anomalous Pulmonary Venous Return \\
EH & Essential Hypertension \\
AF & Atrial Fibrillation \\
LVH & Left Ventricular Hypertrophy \\
CPVT & Catecholaminergic Polymorphic Ventricular Tachycardia \\
CHD & Congenital Heart Disease \\
ARVC & Arrhythmogenic Right Ventricular Cardiomyopathy \\
\hline
\end{tabular}


Figure 4. Disease associations of hCM-enriched genes. (A) Gene-disease network of hCM-enriched genes and cardiovascular diseases. Networks were generated by DisGeNET Cytoscape plugin. Nodes represent genes that showed association with cardiovascular diseases, and edges represent the relationship between linked genes and diseases. (B) List of cardiovascular disease-associated mutations in the top hCMenriched genes. Among the top 50 expressed genes, the ones with known disease-related mutations were listed.

et al., 2013). In contrast, a group of cardiac TFs display similar low promoter $\mathrm{mCpG}$ levels in all three linages we tested. These observations are also in line with the idea that these developmental-related genes are more likely to locate in large genomic domains devoid of DNA methylation in most lineages (Xie et al., 2013). Interestingly, these cardiac-specific TFs were previously found to have high levels of the repressive histone mark $\mathrm{H} 3 \mathrm{~K} 27$ me 3 in hESCs and many other non-cardiac lineages. However, during hESC differentiation towards hCM, the levels of H3K27me3 were decreased in hCM specific TFs, highlighting this mark as a major repressing factor in non-cardiac cell types (Paige et al., 2012; Xie et al., 2013). Whether DNA methylation offers a more stable regulation for cardiac-structural genes expression and whether the highly flexible nature of histone modifications is required for the dynamic cardiac-TF expression in a time-sensitive manner will be interesting questions to ask in future studies.

Overall, our analysis provides new insights into the mechanisms of hCM transcriptional regulation, and stands as an informative and rich resource for investigating cardiac gene functions, as well as providing a better understanding of how the epigenetic machinery coordinates to regulate gene expression in different cell types. Furthermore, the cardiac-specific molecular hallmarks and epigenetic signatures presented in this study might be of relevance for clinical applications as biomarkers for diagnosis and treatment of heart-related diseases.

\section{MATERIALS AND METHODS}

Cell culture and differentiation

H9 hESCs were purchased from WiCell Research, and cultured under standard hESC culture conditions as previously described (Liu et al., 2011). Differentiation of H9 hESC into hNSC was followed by the protocol described previously (Liu et al., 2012).

The hCM derivation from $\mathrm{H} 9 \mathrm{hESC}$ was performed as previously described (Lian et al., 2012), with important modification to further improve the purity. Briefly, single cell suspension of H9 hESCs were seeded onto Matrigel (BD Biosciences) pre-coated cell culture dishes at a density of 100,000 cells per $\mathrm{cm}^{2}$ in mTeSR (StemCell Technologies) in the presence of ROCK inhibitor Y-27632 (Sigma-Aldrich) for $24 \mathrm{~h}$. Cells were then cultured in mTeSR for another 2 days. Differentiation was initiated by treatment with $12 \mu \mathrm{mol} / \mathrm{L}$ CHIR99021 (Selleck) in RPMI/B27-insulin (Life Technologies) for $24 \mathrm{~h}$ (day 0 to day 1), and medium was then changed to RPMI/B27-insulin. On day
3, $5 \mu \mathrm{mol} / \mathrm{L}$ Wnt inhibitor IWP4 (Stemgent) was added into RPMI/ B27-insulin and cells were cultured without medium change for $48 \mathrm{~h}$. From day 5, cells were maintained in RPMI/B27 with medium change every 2-3 days. On day 15 , contracting hCM sheets or clusters were collected by intensive mechanic washing or manually picking, re-plated onto fresh Matrigel pre-coated dishes and maintained in RPMI/B27.

\section{Immunofluorescence}

Cells were fixed with $4 \%$ paraformaldehyde, permeabilized in $0.3 \%$ Triton X-100/PBS and incubated with primary antibodies overnight at $4^{\circ} \mathrm{C}$ at following dilutions: mouse anti-cTnT (1:200, Lab Vision), mouse anti-MF-20 (1:20 Developmental Studies Hybridoma Bank), rabbit anti-MLC2v (1:200, ProteinTech Group), mouse anti-alphaActinin (1:200, Sigma-Aldrich). Cells were then washed and incubated with Alex Fluor 488 goat anti-mouse IgG (1:500, Life Technologies) and/or Alex Fluor 568 goat anti-rabbit $\lg$ (1:500, Life Technologies) for $1 \mathrm{~h}$ at RT. Cell nuclei were counter stained with DAPI ( $1 \mu \mathrm{g} / \mathrm{mL}$, Sigma-Aldrich). Images were captured using a Zeiss LSM780 confocal microscope, and were analyzed in ZEN 2011 software.

\section{Flow cytometry}

Flow cytometry analyses were performed as described previously (Zhang et al., 2012), with antibody concentrations as following: mouse anti-cTnT (1:200, Lab Vision), mouse anti-MF-20 (1:20 Developmental Studies Hybridoma Bank), and Alex Fluor 488 goat anti-mouse IgG (1:500, Life Technologies). Data were collected and analyzed on a LSRII flow cytometer (Becton-Dickinson).

\section{Microarray analysis}

hESC, hNSC, and hCM samples were prepared in biological triplicates. Total RNA was extracted using Trizol Reagent (Life Technologies) and further purified by RNeasy Mini Kit (Qiagen). Microarray experiments were performed using Affymetrix GeneChip PrimeView Human Gene Expression Arrays in the Functional Genomics Core Facility at the Salk Institute for Biological Studies according to the manufacturer's protocol (Affymetrix, Santa Clara, CA). Expression signals were scanned on Affymetrix GeneChip Scanner 3000 7G. Gene expression values were calculated and normalized with RMA using R/Bioconductor. Expression levels were clustered using Cluster 3.0 and visualized using Java TreeView. All data have been deposited in NCBl's Gene Expression Omnibus and are accessible through GEO Series accession number GSE48257. http://www.ncbi.nlm.nih.gov/geo/query/acc.cgi?token= fxqpxgigmoaggzg\&acc $=$ GSE 48257

\section{Quantitative RT-PCR}

RT-qPCR was performed as described previously (Liu et al., 2011). The primer sequences were listed in Table S24.

\section{Genome-wide DNA methylation analysis}

Bisulfite padlock probe sequencing was performed as previously described (Diep et al., 2012). Briefly, genomic DNA was extracted 
A

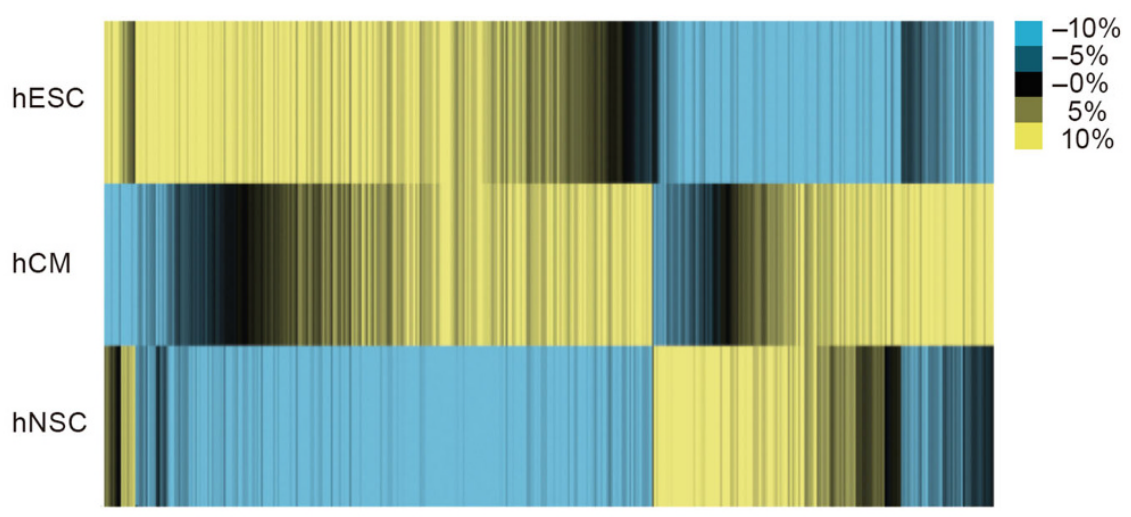

B

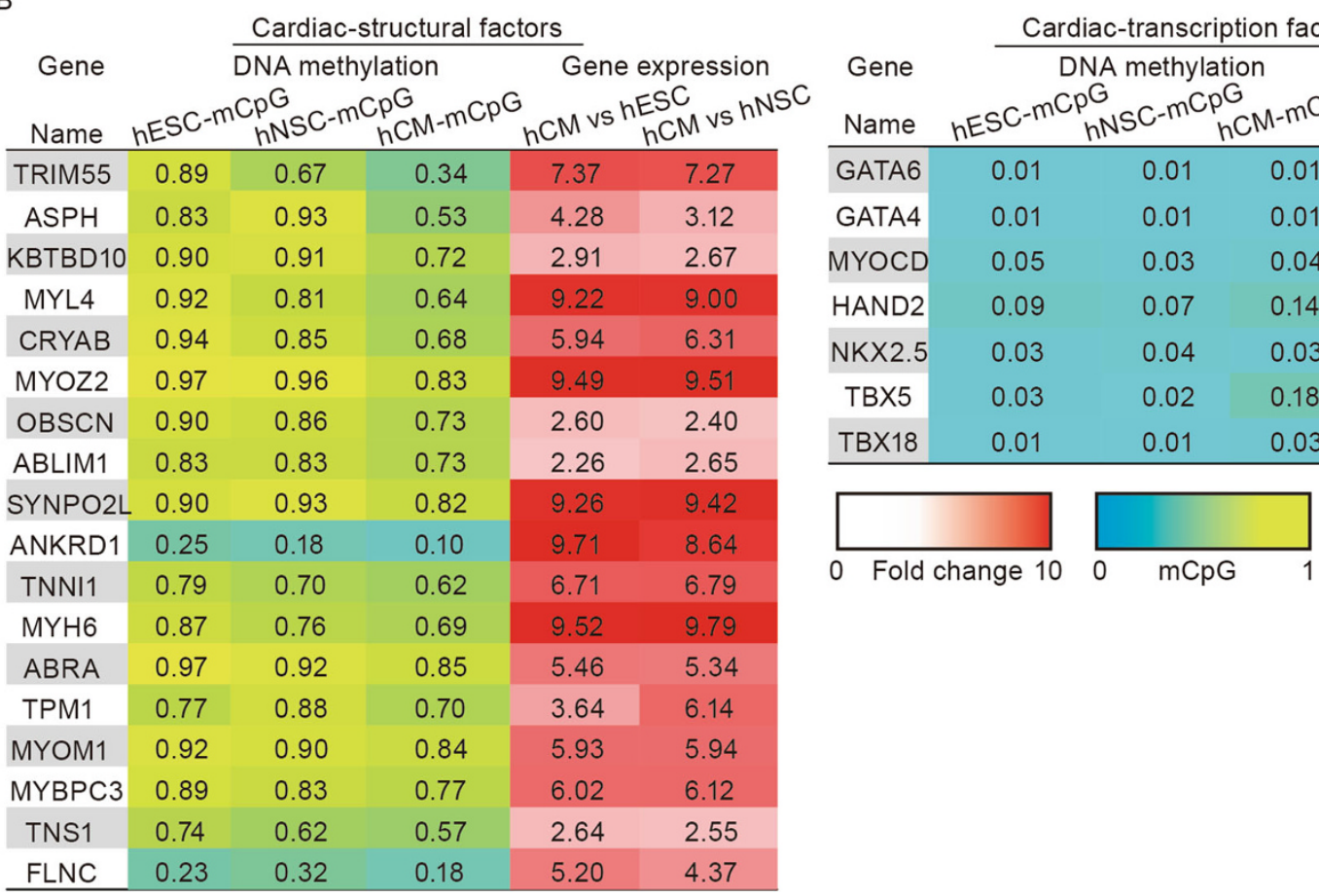

Figure 5. Genome-wide DNA methylation analysis of hCMs. (A) Heatmap and hierarchical clustering of CpG methylation levels measured within $1 \mathrm{~kb}$ of promoter regions. Color represents the $\mathrm{mCpG} / \mathrm{CpG}$ level relative to mean. (B) List of cardiac-structural factors and cardiac-transcription factors with their $\mathrm{mCpG}$ levels and relative fold changes in their gene expression.

from H9-hESC, H9-hNSC, and H9-hCM using QIAamp DNA Micro Kit (QIAGEN), and approximately $1000 \mathrm{ng}$ of genomic DNA was bisulfite converted with EZ-96 DNA Methylation-Lightning MagPrep kit (Zymo Research). Approximately $250 \mathrm{ng}$ of bisulfite converted genomic DNAs were mixed with normalized amount of the genomewide scale padlock probe set. The annealed padlock probes were polymerized, gap-filled, and ligated to generate circularized DNA. The circularized captured targets were amplified and barcoded by PCR using the library-free BSPP protocol as previously described (Diep et al., 2012). The resulting bisulfite sequencing libraries were pooled in the same molar ratio, size-selected at the fragment size approximately $375 \mathrm{bp}$ in 6\% TBE PAGE gel (Life Technologies), and sequenced by Illumina HiSeq2000 sequencer (110 bp, paired-end reads). The bisulfite reads were mapped to the in silico bisulfite- converted human genome sequences (hg19) by bisREADMapper (Diep et al., 2012). DNA methylation frequency (at level from 0-1) at each CpG site with minimum 10x depth coverage was calculated. Only $\mathrm{CpG}$ sites with 10x coverage in each cell type were used for differential analysis. Promoter $\mathrm{CpG}$ methylation levels were calculated by averaging the $\mathrm{mCpG} / \mathrm{CpG}$ ratio for all $\mathrm{CpG}$ dinucleotides with $10 \times$ coverage within $1 \mathrm{~kb}$ of the TSS. Clustering of promoter methylation ratios was carried out using Cluster 3.0 and visualized using Java TreeView.

\section{Gene Ontology (GO) analysis}

Functional properties of the differentially expressed or methylated genes were categorized using the BiNGO Cytoscape plugin as 
described previously (Maere et al., 2005). A $P$-value of less than 0.05 was considered statistically significant.

\section{Gene regulatory networks reconstruction}

Differentially expressed genes obtained after the performance of a $t$-test with a $P$-value $<0.05$ were connected to expression regulatory interactions from literature. For this specific purpose we use the information contained in the ResNet mammalian database from Ariadne Genomics (http://www.ariadnegenomics.com/) (Novichkova et al., 2003; Daraselia et al., 2004). We selected only the interactions included in the category of Promoter Binding and Direct Regulation. In order to contextualize the network to the biological conditions under which the expression data was obtained we applied an algorithm that exploits the consistency between predicted and known stable states from experimental data to guide an iterative network pruning. The algorithm predicted missing expression values in gene regulatory networks, and could be applied to contextualize the network when all the expression values in two attractors are known. The method assumes a Boolean model to compute attractors of networks that are iteratively pruned by means of an evolutionary algorithm. The evolutionary algorithm samples the probability distribution of positive circuits and individual interactions within the subpopulation of the best-pruned networks at each iteration. The resulting contextualized network is based not only on previous knowledge about local connectivity but also on a global network property (stability).

Attractor computation was performed assuming a Boolean model applying a synchronous updating scheme (Garg et al., 2008) that updates all gene states simultaneously at each step until the system reaches an attractor. For this purpose we used our own implementation (Crespo et al., 2013) written in Perl of the algorithm described by Garg and co-workers (Garg et al., 2007). We implemented the Johnsons algorithm (Johnson, 1975) to detect all elementary circuits in the network. Both elementary circuit detection and positive circuits sorting scripts were implemented in Perl.

\section{Gene functional association network analysis}

Networks of gene associations (co-expression, co-localization, genetic interaction, pathway, and more) were generated using the GeneMANIA Cytoscape plugin as described previously (Montojo et al., 2010). The "query gene based" weighting method was used in this study. Sub-networks were generated by selecting genes that were categorized in the same functional GO group.

\section{Gene-disease association network analysis}

Network of gene-disease associations (altered expression, casual mutation, genetic variation, marker, and more) were generated using DisGeNET Cytoscape plugin as described previously (Bauer-Mehren et al., 2010). The top $50 \mathrm{hCM}$ up-regulated genes were inquired individually for disease association in the cardiovascular disease class. All the individual gene-disease networks were then combined based on the shared disease type.

\section{ACKNOWLEDGEMENTS}

We thank Dr. Yue Ma for helpful discussion. J.Q. is supported by the National Basic Research Program (973 Program) (No. 2014CB910500).
G.H.L. is supported by the Strategic Priority Research Program of the Chinese Academy of Sciences (XDA01020312), the National Basic Research Program of China (973 Program) (No. 2014CB964600), the National Natural Science Foundation (Grant Nos. 81271266 and 31222039), the National Laboratory of Biomacromolecules, and the State Key Laboratory of Drug Research (SIMM1302KF-17). K.S. and M.L. are supported by a CIRM training grant. K.Z is supported by California Institute for Regenerative Medicine (RB3-05083) and NIH (R01 GM097253). J.C.I.B is supported by TERCEL-ISCIII-MINECO, CIBER-BBN, Cardiocel, Fundacion Cellex, G. Harold and Leila Y. Mathers Charitable Foundation, The Leona M. and Harry B. Helmsley Charitable Trust and The Ellison Medical Foundation.

\section{ABBREVIATIONS}

cTnT, cardiac troponin T; GO, Gene Ontology; hESCs, human embryonic stem cells; hCM, human cardiomyocyte; hNSCs, hESCderived neural stem cells; TFs, transcription factors.

\section{COMPLIANCE WITH ETHICS GUIDELINES}

Ying Gu, Guang-Hui Liu, Nongluk Plongthongkum, Christopher Benner, Fei Yi, Jing Qu, Keiichiro Suzuki, Jiping Yang, Weiqi Zhang, Mo Li, Nuria Montserrat, Isaac Crespo, Antonio del Sol, Concepcion Rodriguez Esteban, Kun Zhang and Juan Carlos Izpisua Belmonte declare that they have no conflict of interest.

This article does not contain any studies with human or animal subjects performed by the any of the authors.

\section{OPEN ACCESS}

This article is distributed under the terms of the Creative Commons Attribution License which permits any use, distribution, and reproduction in any medium, provided the original author(s) and the source are credited.

\section{REFERENCES}

Bauer-Mehren A, Rautschka M, Sanz F, Furlong LI (2010) DisGeNET: a Cytoscape plugin to visualize, integrate, search and analyze gene-disease networks. Bioinformatics 26:2924-2926

Beqqali A, Kloots J, Ward-van Oostwaard D, Mummery C, Passier R (2006) Genome-wide transcriptional profiling of human embryonic stem cells differentiating to cardiomyocytes. Stem Cells 24:1956-1967

Cao F, Wagner RA, Wilson KD, Xie X, Fu JD, Drukker M, Lee A, Li RA, Gambhir SS, Weissman IL et al (2008) Transcriptional and functional profiling of human embryonic stem cell-derived cardiomyocytes. PLoS One 3:e3474

Crespo I, Krishna A, Le Bechec A, del Sol A (2013) Predicting missing expression values in gene regulatory networks using a discrete logic modeling optimization guided by network stable states. Nucleic Acids Res 41:e8

Daraselia N, Yuryev A, Egorov S, Novichkova S, Nikitin A, Mazo I (2004) Extracting human protein interactions from MEDLINE using a full-sentence parser. Bioinformatics 20:604-611

Diep D, Plongthongkum N, Gore A, Fung HL, Shoemaker R, Zhang K (2012) Library-free methylation sequencing with bisulfite padlock probes. Nat Methods 9:270-272 
Garg A, Xenarios I, Mendoza L, DeMicheli G (2007) Lecture notes in computer science vol. 4453. In: Speed T, Huang $\mathrm{H}$ (eds). Springer, Berlin, p 62-76

Garg A, Di Cara A, Xenarios I, Mendoza L, De Micheli G (2008) Synchronous versus asynchronous modeling of gene regulatory networks. Bioinformatics 24:1917-1925

Johnson DB (1975) Finding all the elementary circuits of a directed graph. SIAM J Comput 4:77-84

Kattman SJ, Witty AD, Gagliardi M, Dubois NC, Niapour M, Hotta A, Ellis J, Keller G (2011) Stage-specific optimization of activin/nodal and BMP signaling promotes cardiac differentiation of mouse and human pluripotent stem cell lines. Cell Stem Cell 8:228-240

Lian X, Hsiao C, Wilson G, Zhu K, Hazeltine LB, Azarin SM, Raval KK, Zhang J, Kamp TJ, Palecek SP (2012) Robust cardiomyocyte differentiation from human pluripotent stem cells via temporal modulation of canonical Wnt signaling. Proc Natl Acad Sci USA 109:E1848-E1857

Liu GH, Barkho BZ, Ruiz S, Diep D, Qu J, Yang SL, Panopoulos AD, Suzuki K, Kurian L, Walsh C et al (2011) Recapitulation of premature ageing with iPSCs from Hutchinson-Gilford progeria syndrome. Nature 472:221-225

Liu GH, Qu J, Suzuki K, Nivet E, Li M, Montserrat N, Yi F, Xu X, Ruiz S, Zhang $W$ et al (2012) Progressive degeneration of human neural stem cells caused by pathogenic LRRK2. Nature 491:603-607

Maere S, Heymans K, Kuiper M (2005) BiNGO: a Cytoscape plugin to assess overrepresentation of gene ontology categories in biological networks. Bioinformatics 21:3448-3449

Montojo J, Zuberi K, Rodriguez H, Kazi F, Wright G, Donaldson SL, Morris Q, Bader GD (2010) GeneMANIA Cytoscape plugin: fast gene function predictions on the desktop. Bioinformatics 26: 2927-2928

Novichkova S, Egorov S, Daraselia N (2003) MedScan, a natural language processing engine for MEDLINE abstracts. Bioinformatics 19:1699-1706
Paige SL, Thomas S, Stoick-Cooper CL, Wang H, Maves L, Sandstrom R, Pabon L, Reinecke H, Pratt G, Keller G et al (2012) A temporal chromatin signature in human embryonic stem cells identifies regulators of cardiac development. Cell 151:221232

Shannon P, Markiel A, Ozier O, Baliga NS, Wang JT, Ramage D, Amin N, Schwikowski B, Ideker T (2003) Cytoscape: a software environment for integrated models of biomolecular interaction networks. Genome Res 13:2498-2504

Synnergren J, Akesson K, Dahlenborg K, Vidarsson H, Ameen C, Steel D, Lindahl A, Olsson B, Sartipy P (2008) Molecular signature of cardiomyocyte clusters derived from human embryonic stem cells. Stem Cells 26:1831-1840

Willems E, Cabral-Teixeira J, Schade D, Cai W, Reeves P, Bushway PJ, Lanier M, Walsh C, Kirchhausen T, Izpisua Belmonte JC et al (2012) Small molecule-mediated TGF-beta type II receptor degradation promotes cardiomyogenesis in embryonic stem cells. Cell Stem Cell 11:242-252

Xie W, Schultz MD, Lister R, Hou Z, Rajagopal N, Ray P, Whitaker JW, Tian S, Hawkins RD, Leung D et al (2013) Epigenomic analysis of multilineage differentiation of human embryonic stem cells. Cell 153:1134-1148

Yang L, Soonpaa MH, Adler ED, Roepke TK, Kattman SJ, Kennedy M, Henckaerts E, Bonham K, Abbott GW, Linden RM et al (2008) Human cardiovascular progenitor cells develop from a KDR+ embryonic-stem-cell-derived population. Nature 453:524-528

Zhang J, Klos M, Wilson GF, Herman AM, Lian X, Raval KK, Barron MR, Hou L, Soerens AG, Yu J et al (2012) Extracellular matrix promotes highly efficient cardiac differentiation of human pluripotent stem cells: the matrix sandwich method. Circ Res 111:1125-1136 